\title{
First trimester fetal heart evaluation. A pictorial essay
}

\author{
Ioana Cristina Rotar¹, Daniel Mureșan¹, Claudiu Mărginean², Dominic Gabriel Iliescu ${ }^{3}$, \\ Ştefania Tudorache ${ }^{3}$
}

\begin{abstract}
${ }^{1} 1^{\text {st }}$ Department of Obstetrics and Gynecology, University of Medicine and Pharmacy "Iuliu Hațieganu" Cluj-Napoca, ${ }^{2}$ Department of Obstetrics and Gynecology, University of Medicine, Pharmacy, Sciences and Technology, Târgu Mureș, ${ }^{3}$ Department of Obstetrics and Gynecology, University of Medicine and Pharmacy, Craiova, Romania
\end{abstract}

\begin{abstract}
Classically fetal heart evaluation is mandatory in the second trimester of pregnancy. Recent data suggest that the diagnosis of congenital heart disease is feasible in the first trimester (FT) of pregnancy, especially for trained examiners. In this pictorial essay we aim to illustrate in detail the particularity of the FT heart evaluation: the specific ultrasound techniques including practical tips for the basic and extended first trimester fetal heart evaluation protocol. The diagnosis is possible by the use of a variety of ultrasound techniques (B mode, CFM, Bidirectional Doppler, STIC, TUI) using the transabdominal/transvaginal probes. An abnormal FT heart scan requires second trimester reassessment. Unfortunately, not all cardiac disease can be potentially diagnosed in the first trimester. Based on their own experience the authors recommend that heart examination in the FT should be attempted in all fetuses, even in low risk pregnancies, using at least the basic Doppler examination protocol.

Keywords: fetal heart; congenital heart defects; ultrasound; spatio-temporal image correlation (STIC); four chamber view
\end{abstract}

\section{Introduction}

The obstetrical ultrasound (US) had a fulminant evolution in the latest decades; the number of US visualisable structures in the first trimester (FT) of pregnancy being in a continuous growth. Among them, the confirmation of the normality of the fetal heart (FH) is particularly important due to an increased prevalence of congenital heart defects (CHD); $0.8 \%$ of the births in the European Union, representing $30.47 \%$ of all congenital defects [1].

Fetal cardiac evaluation should be mandatory in the FT in high risk patients: maternal conditions (pregestational diabetes, phenylketonuria, lupus or Sjögren syndrome with positive SSA/SSB autoantibody, exposure to

Received 20.04.2020 Accepted 30.06.2020

Med Ultrason

2021, Vol. 23, No 2, 220-225

Corresponding author: Prof Daniel Mureșan MD, PhD

$1^{\text {st }}$ Department of Obstetrics and Gynecology

University of Medicine and Pharmacy

"Iuliu Hațieganu" Cluj Napoca, Romania

E-mail: muresandanie101@yahoo.com
Carbamazepine, Lithium, Retinoic acid, NSAID), IVF pregnancy, monochorionic twinning or consanguinity [2]. Currently the most frequent referral for an extensive cardiac examination is abnormal ultrasound findings (associated anomalies, increased nuchal translucency, abnormal ductus venosus flow or tricuspid regurgitation in the FT) or chromosomal anomalies [3]. Heart evaluation in low-risk population nowadays is not routinely performed, despite the fact that $90 \%$ of CHD occurs in low risk pregnancies [4].

FT FH evaluation can be challenging due to the small heart dimensions, increased heart frequency, active fetal movements, unfavorable fetal position or well-represented adipose panicle in obese patients. The second trimester (ST) ultrasound detection is the gold standard today, having a proved efficacy and lowering the rate of perioperative mortality [5]. However, an increasing number of evidences suggested that the diagnosis of CHD is also feasible at the end of the FT [6-9].

In this pictorial essay we present in images our experience related to the possibilities of FT US for the diagnostic of CHD together with a short literature review. 


\section{Techniques used for FH scanning in the first trimester}

FH examination in the FT is directly linked to the cardiovascular embryology. A conclusive evaluation of the heart structures is achievable at the end of the FT (11-13 gestational weeks- $\mathrm{GW}+6$ days) [7-9].

The evaluation starts in B mode with the upper abdominal section, an oblique plan through the liver at the level of the umbilical vein, stomach and posteriorly the spine (fig 1a). By a slight cranial shift, the four-chamber view (4CV) can be easily obtained (fig $1 \mathrm{~b}$ ). On $4 \mathrm{CV}$ the following structures can be examined: heart position, axis and dimensions, morphology and dimensions of the two atria and ventricles, ventricular and interatrial septum integrity, atrioventricular valves presence and movement. By cranial movement and slight rotations, the right and left ventricular outflow tract can be obtained.

Color Doppler examination (color flow mode-CFM and/or directional power Doppler) improves the visuali-

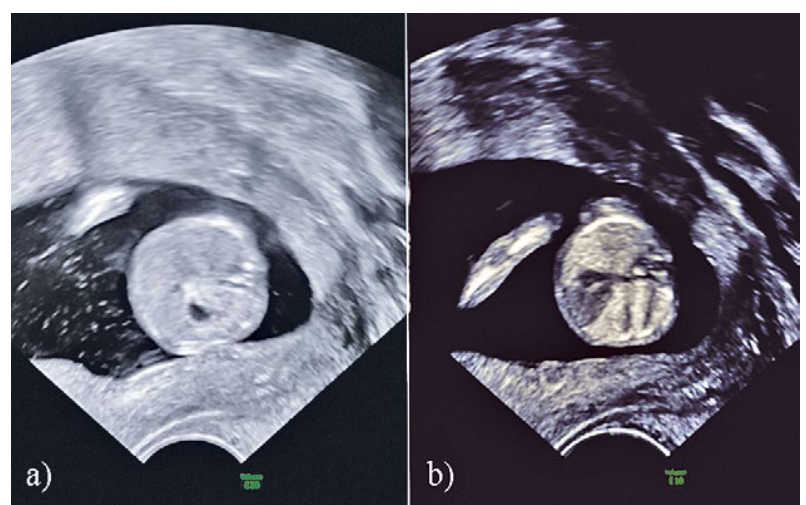

Fig 1. B-mode evaluation of the heart in the first trimester: a) upper abdominal transverse section; b) four-chamber view.

zation of great vessels enhancing CHD detection rates [7]. The simplified Doppler evaluation protocol includes two plans (basic evaluation): the $4 \mathrm{CV}$ and the three vessels and trachea view (3VT) (fig 2a,b). In good scanning conditions, the ductal arch and aortic arch are visible on

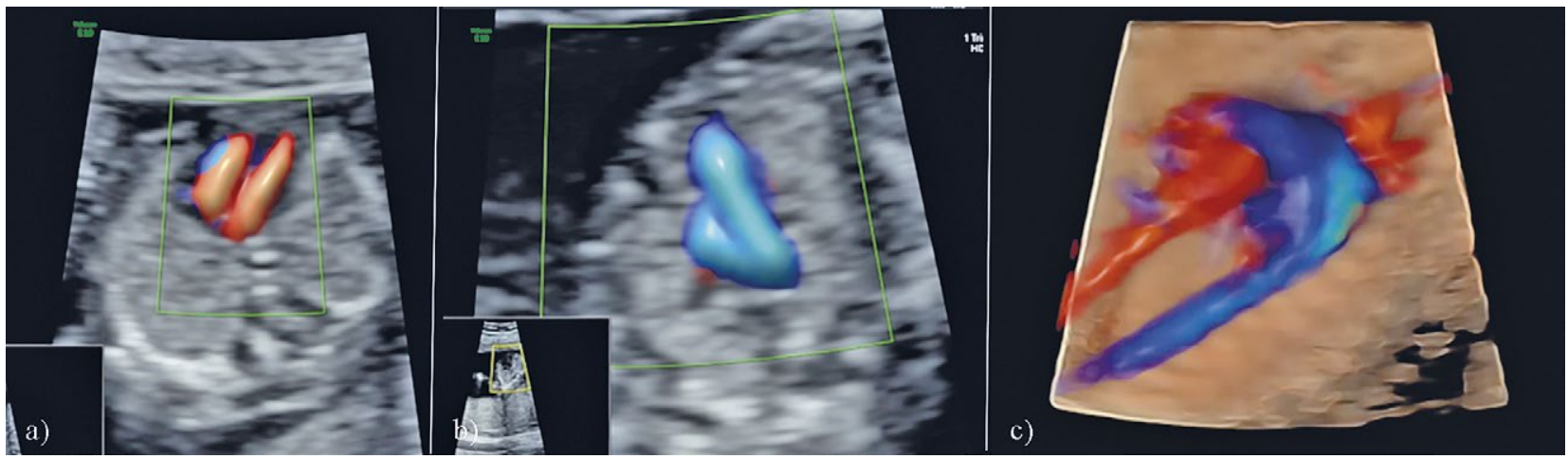

Fig 2. Examination of a normal fetal heart in the first trimester using the CFM transabdominal probe: a) four-chamber view - normal diastolic flow from both right and left atrium into right and respectively left ventricle; b) "V" sign - aortic arch and pulmonary arch; c) parasagittal view of the aortic and ductal arch
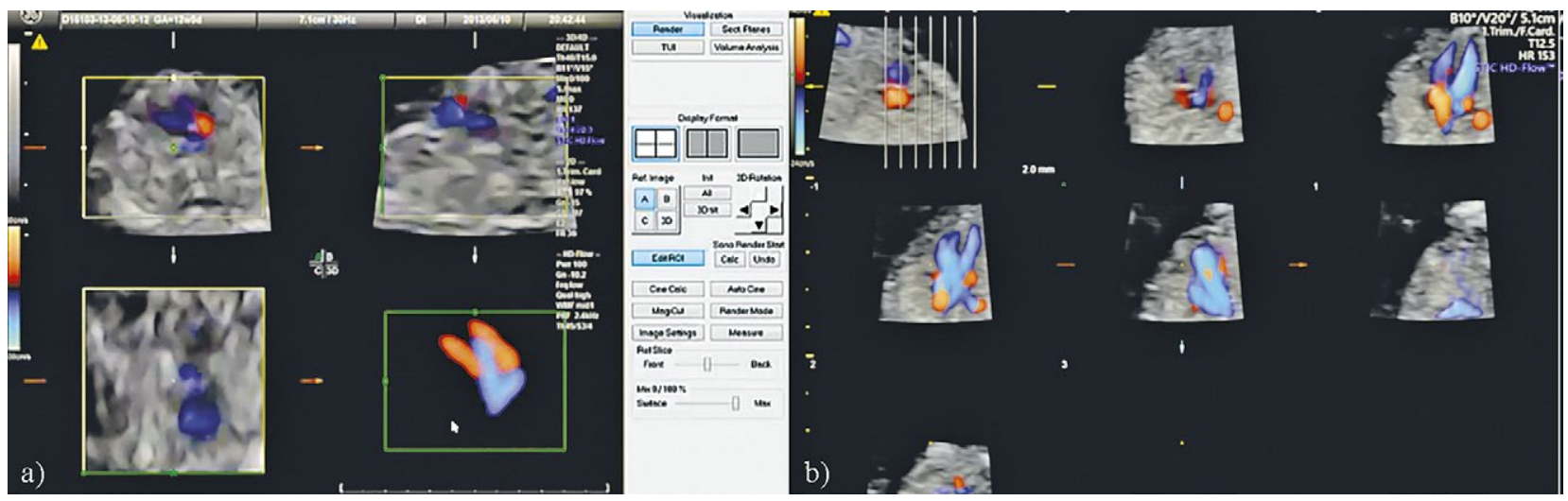

Fig 3. 3D technique for the evaluation of the fetal heart in the first trimester: a) spatiotemporal image correlation (STIC) acquisition with 3D rendered image - diastolic ventricular inflow and systolic aortic and pulmonary flow can be seen on the same image; b) tomographic ultrasound imaging in a STIC acquisition. 
parasagittal views (fig 2c). The visualization of systemic venous drainage, more difficult, should be attempted in the extending scanning protocol.

The 3D/4D techniques ameliorates heart structures visualization. Moreover, it allows the analysis of the cardiac cycle. The spatiotemporal image correlation software (STIC) improves the accuracy of the evaluation of the FH in the FT providing a better resolution (fig 3a) $[9,10]$. Furthermore, the use of tomographic ultrasound imaging (TUI) displays several cross-sectional images in three orthogonal planes and at specific distances from 4CV (fig 3b) [10].

For screening purposes, the transabdominal (TA) approach is preferred. The transvaginal examination (TV) is particularly useful in obese patients or in retroverted uterus (fig 4).

The complete evaluation of FH in the FT should include evaluation of the cardiac situs, cardiac connections, atrioventricular junction, right-left side symmetry and septal-aortic continuity [10]. The visualization and normality of all of the above-mentioned structures allows an early certification of a normal heart in the FT. In some cases, it is difficult to obtain all these plans by $2 \mathrm{D}$ ultrasound. Instead, the simplified protocol using together $2 \mathrm{D}$, CFM and STIC with two planes $(4 \mathrm{CV}, 3 \mathrm{CV})$ is feasible for the routine use in the FT [7-9].

\section{Abnormal heart in the first trimester}

Unfortunately, not all CHD can be diagnosed in the FT of pregnancy; exceptions - evolving (eg: pulmonary stenosis, aortic coarctation) or late onset cardiac diseases (eg. fibroelastosis). Based on the literature [7-12] and on our own experience, CHD in the FT can be divided in easy detectable and difficult or impossible to detect (table I).

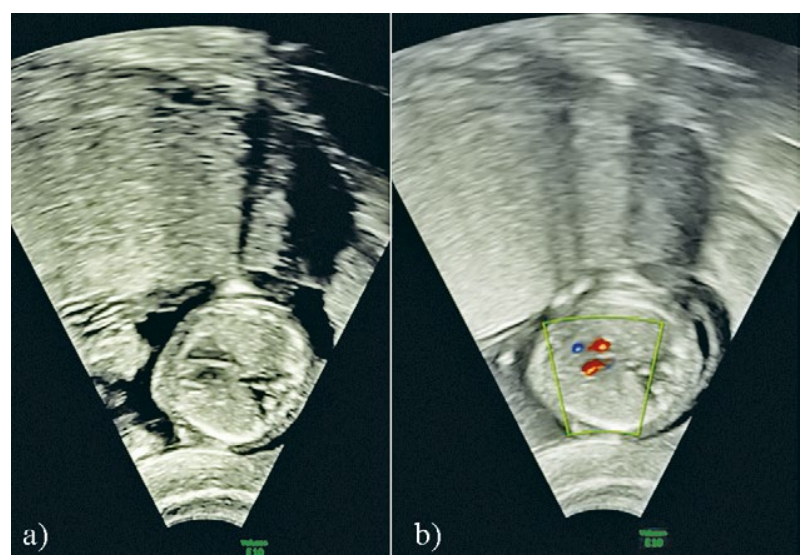

Fig 4. Transvaginal four-chamber view section at 12 weeks of gestation: a) B mode- four-chamber view; b) CFM - fourchamber view

In the following sections, we aim to exemplify that an ultrasound diagnosis of CHD in the FT can be sustained. In most of the cases, a FT CHD suspicion was reevaluated in second trimester when the final prenatal diagnosis was established. The earlier the diagnosis, the better the prognosis $[5,12]$.

\section{Dextrocardia}

In the figure $5 \mathrm{a}$ the stomach is located on the left side of the abdomen. At the level of $4 \mathrm{CV}$ the apex of the heart is pointing to the right side of the fetus, a condition known as dextrocardia (fig 5b). The $4 \mathrm{CV}$ in this case looks completely normal. The outflow tracts have also a normal appearance (fig $5 \mathrm{c}, \mathrm{d}$ ).

Ventricular septal defects (VSD) are the second most common CHD. In figure 6 the B mode (a) revealed an interruption, anechoic area, in the cranial part of the ventricular septum. The CFM (b) shows a clear flow through the septum during the ventricular systole, probably an in-

Table I. Classification of congenital heart defects based on first trimester detectability

\begin{tabular}{lc}
\hline Easy detectable & Difficult or impossible to detect \\
\hline - hypoplastic hearts (left or right - tricuspid atresia) & - tetralogy of Fallot without severe pulmonary stenosis \\
- single ventricle & - partial AVSDs \\
- isolated dextrocardia & - aortic coarctation \\
- heterotaxy syndromes & - interrupted aortic arch \\
- common arterial trunk & - obstructive disease of semilunar valves (aortic and pulmonary stenosis) \\
- double outlet right ventricle & - corrected great arteries transposition \\
- double inlet ventricle & - ventricular septal defects \\
- uncorrected great arteries transpositions & - venous abnormalities: \\
- complete atrioventricular septal defects & $\bullet$ persistent left superior vena cava \\
- pulmonary atresia with intact septum & $\bullet$ interrupted inferior vena cava \\
- right-sided aortic arches abnormalities & $\bullet$ total/partial anomalous pulmonary venous return \\
& - evolving diseases: \\
& $\bullet$ tumors \\
& $\bullet$ Ebstein diseasew \\
& $\bullet$ fibroelastosis \\
\hline
\end{tabular}




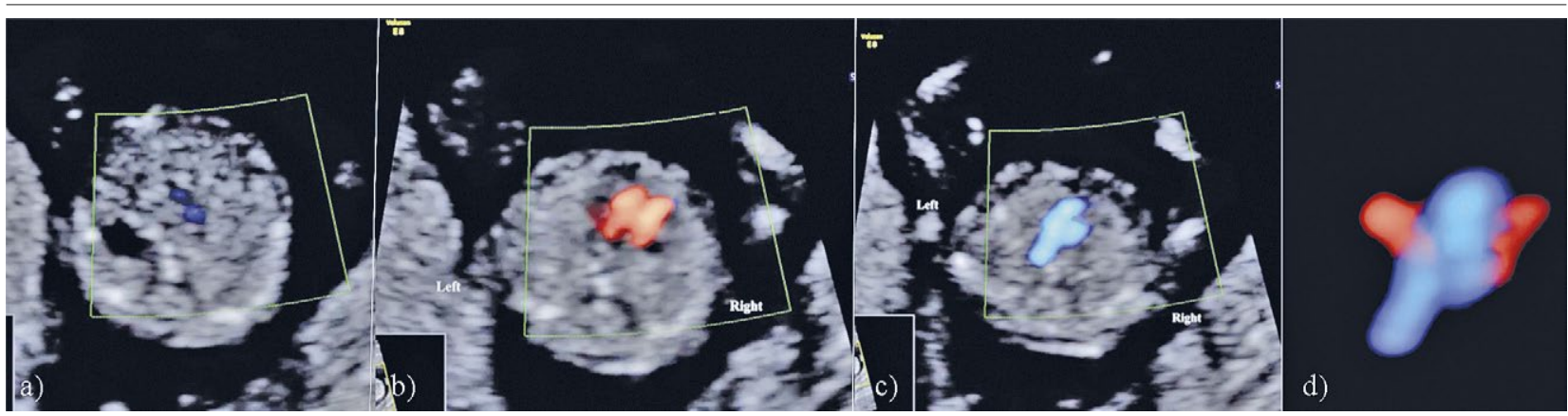

Fig 5. Dextrocardia CFM mode: a) upper abdomen section - stomach on the lef, umbilical vein (blue) in the middle of the image; b) four-chamber view - normal diastolic flow in atria and ventricles with the apex of the heart pointinig to the right; c) outflow tracts; d) $3 \mathrm{D}$ rendering acquisition of the outflow tracts

let VSD. VSD in the first trimester are difficult to detect due to the small dimensions of the defect [12]. They are rarely isolated, more frequently being encountered in the case of complex cardiac malformations.

Atrioventricular septal defect (AVSD) is a CHD determined by an insufficient septation of the heart associated with anomalies of the AV valves. In figure 7 a complete form of AV valve is presented: single ventricular inflow though the unique atrioventricular valve towards both ventricles. The genetic evaluation of fetuses with AVSD is mandatory; an association with Down syndrome or trisomy 18 is frequently encountered especially when a high nuchal translucency is detected.

An early morphologic evaluation of the ventricles in the FT is possible. In figure 8 a a ventricular disproportion can be seen in 4CV: a smaller left ventricle compared to a normal right ventricle. In this case, the second trimester follow-up revealed an unbalanced partial atrioventricular septal defect with aortic arch hypoplasia. Usually the hypoplastic left heart syndrome is due to the underdevelopment of the left ventricle secondary to the

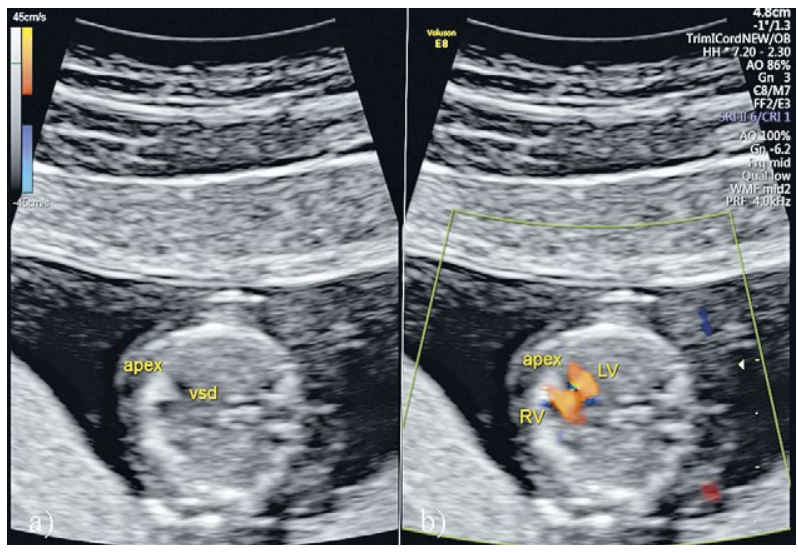

Fig 6. Ventricular septal defects (vsd): a) four-chamber view B mode; b) four-chamber view - CFM. RV, right ventricle; LV, left ventricle. severe stenosis of the mitral and/or aortic valve or aortic coarctation with no/little communication between the left ventricle and the aorta [12]. Less frequent the right

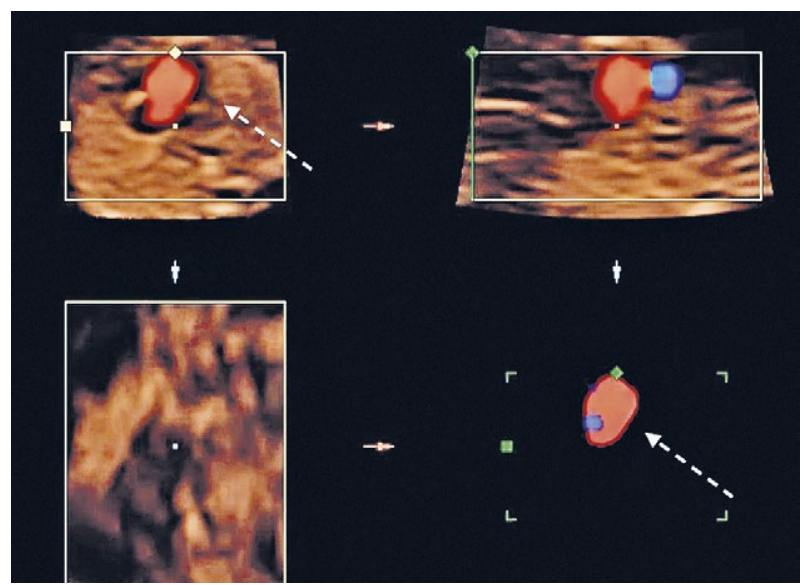

Fig 7. Atrioventricular septal defect: spatiotemporal image correlation acquisition CFM diastolic flow, in four-chamber view and rendered image of the flow

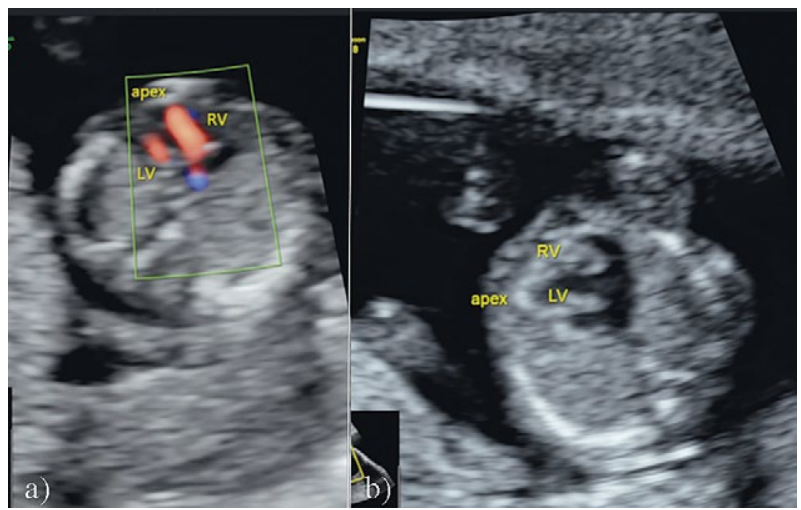

Fig 8. Ventricular disproportion: a) four-chamber view CFM small left ventricle, normal right ventricle; b) axial grayscale four-chamber view - apex and small right ventricle (RV), large left ventricle (LV). 


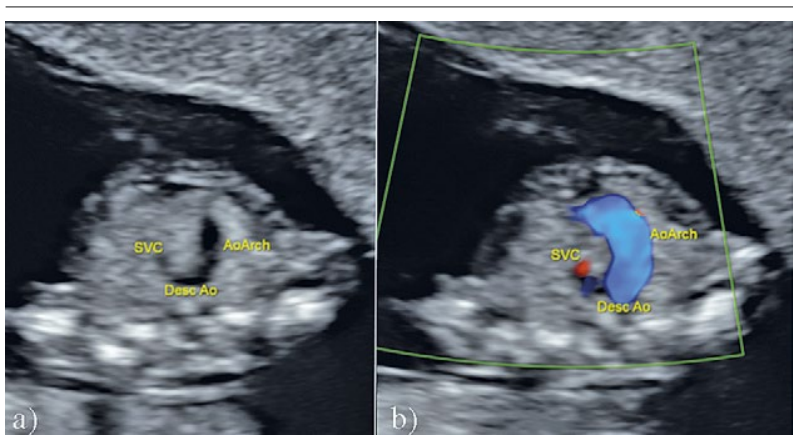

Fig 9. Abnormal three-vessel view in a fetus with complex congenital heart defects with hypoplastic ductal arch: a) B mode; b) CFM. SVC, superior vena cava; DescAo, descending aorta; Ao arch, aortic arch.

ventricle can be hypoplastic in the case of tricuspid atresia (fig $8 \mathrm{~b}$ ) or pulmonary stenosis. In the B mode at the $4 \mathrm{CV}$ the right ventricle is obviously smaller than the left ventricle.

The assessment of the great vessels is an essential step. Anomalies of the great vessels can potentially be diagnosed in the FT of pregnancy. In figure 9, a complex case of CHD is demonstrated - a fetus with severe tricuspid atresia and small right ventricle. In the three-ves- sel view (3VV) only two vessels are seen: superior vena cava and the aorta, the pulmonary artery not being visible nor in B mode (fig 9a), nor in CFM (fig 9b). The 3VV was essential for the diagnosis.

Aberrant right subclavian artery (ARSA) represent an anatomic variant when the subclavian artery arises directly from the aortic arch instead of the brachiocephalic trunk, more frequently encountered in Down syndrome compared to euploid fetuses (fig 10) [12].

Color Doppler allows a good evaluation of the ventriculoarterial connections, essential for the diagnosis of transposition of great vessel (fig 11).

\section{The performance of ultrasound scan between 11-14 WA}

The visualization rates of fetal cardiac structures are higher towards the end of FT. Accordingly, CHD detection rates varies from $20 \%$ at $11 \mathrm{WG}$ up to $92 \%$ at 13 WG [13].

In Quarello et al study, the visualization rate of $4 \mathrm{CV}$ was $86 \%$ but 3 VT was evaluated only in $79 \%$ of fetuses [8]. The visualization rates of $4 \mathrm{CV}$ was not influenced by the gestational age, but $3 \mathrm{VT}$ view was better seen

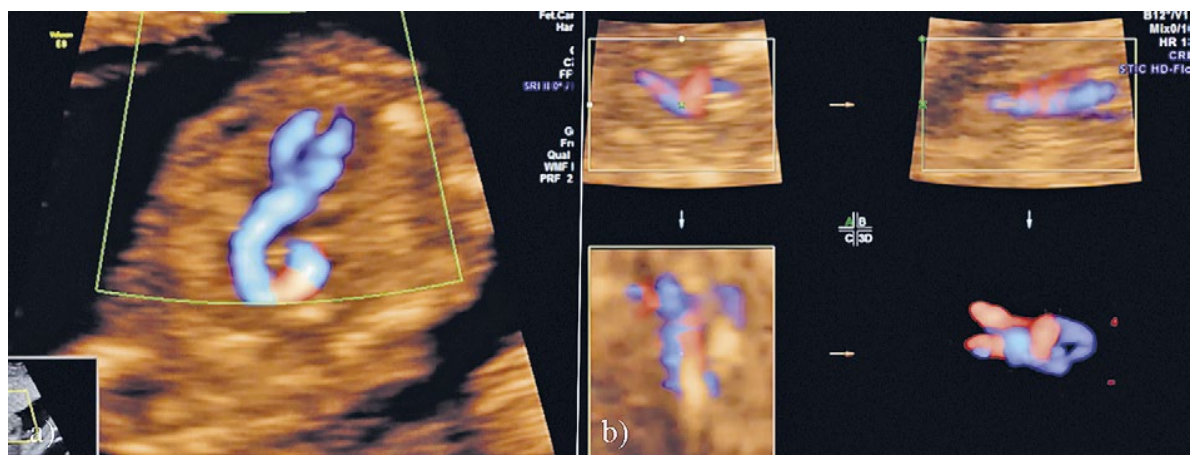

Fig 10. Aberrant right subclavian artery: a) retrotracheal route of the right subclavian artery; b) orthogonal acquisition with $3 \mathrm{D}$ rendering

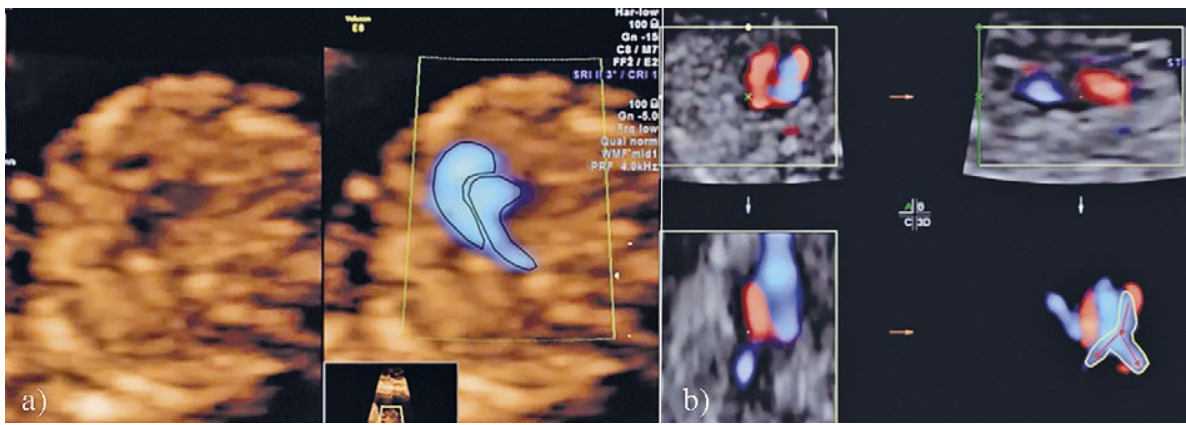

Fig 11. Transposition of the great vessels: a) aorta and pulmonary artery have a parallel route at the emergence from the ventricles. There is not the normal crossing; b) 3D acquisition and rendered image shows that the artery that arise from the left ventricle has a typical pulmonary bifurcation, confirming the diagnostic of transposition. 
when the CRL was $>75 \mathrm{~mm}[8]$. The use of $4 \mathrm{CV}$ section alone had a sensitivity of only $45.71 \%$ compared to the $4 \mathrm{CV}+3 \mathrm{VT}-88.57 \%$ for the detection of CHD [7].

The use of transvaginal probes provides a higher resolution particularly suitable for obese women or in the case of a retroverted uterus. Indeed, in $58 \%-62 \%$ of cases the use of a 5-9 MHz TV probe obtains a satisfactory assessment of the FH structures, the visualization rates increasing linearly with the gestational age [14]. In a cross-sectional study that compared TA versus TV US, the latter was usually superior before 14 GWs [14].

The early detection rates of major CHD depend not only on the protocol used, but also on the examiner skills; ranging in an unselected population between $2.3-56 \%$ [15]. In contrast, in high-risk populations, cardiac examination reached a sensitivity of $78.5-90 \%$ with a $74.5 \%$ in concordance with the second trimester diagnosis [15].

The use of STIC ameliorates the intra- and interobserver agreement $[9,11]$.

\section{Conclusions}

The capacity to achieve good quality cardiac ultrasound images, important for the confirmation of the normality of fetal heart in the FT, is feasible and depends not only on ultrasound probes and software, but, more important, on the sonographer's experience, commitment and awareness. New techniques of ultrasound - Doppler and 3/4D methods - greatly improve the diagnostic capabilities. The heart is a developing organ; therefore, even after a successfully complete heart scan in the FT, the heart should be reassessed in every trimester due the potential evolution of the cardiac condition.

\section{Conflict of interest: none}

\section{References}

1. European Commission. Prevalence charts and tables. Available at: https://eu-rd-platform.jrc.ec.europa.eu/eurocat/ eurocat-data/prevalence_en. Accessed on 12.04.2020.

2. Donofrio MT, Moon-Grady AJ, Hornberger LK, et al. Diagnosis and treatment of fetal cardiac disease: a scientific statement from the American Heart Association. Circulation 2014;129:2183-2242.

3. AIUM Practice Parameter for the Performance of Fetal Echocardiography. J Ultrasound Med 2020;39:E5-E16.
4. Bishop KC, Kuller JA, Boyd BK, Rhee EH, Miller S, Barker P. Ultrasound examination of the fetal heart. Obstet Gynecol Surv 2017;72:54-61.

5. Li YF, Zhou KY, Fang J, Wang C, Hua YM, Mu DZ. Efficacy of prenatal diagnosis of major congenital heart disease on perinatal management and perioperative mortality: a meta-analysis. World J Pediatr 2016;12:298307.

6. García Fernández S, Arenas Ramirez J, Otero Chouza MT, Rodriguez-Vijande Alonso B, Llaneza Coto AP. Early fetal ultrasound screening for major congenital heart defects without Doppler. Eur J Obstet Gynecol Reprod Biol 2019;233:93-97.

7. Wiechec M, Knafel A, Nocun A. Prenatal detection of congenital heart defects at the 11- to 13-week scan using a simple color Doppler protocol including the 4-chamber and 3-vessel and trachea views. J Ultrasound Med 2015;34:585-594.

8. Quarello E, Lafouge A, Fries N, Salomon LJ, CFEF. Basic heart examination: feasibility study of first-trimester systematic simplified fetal echocardiography. Ultrasound Obstet Gynecol 2017;49:224-230.

9. Tudorache S, Cara M, Iliescu DG, Novac L, Cernea N. First trimester two- and four-dimensional cardiac scan: intraand interobserver agreement, comparison between methods and benefits of color Doppler technique. Ultrasound Obstet Gynecol 2013;42:659-668.

10. Hernandez-Andrade E, Patwardhan M, Cruz-Lemini M, Luewan S. Early Evaluation of the Fetal Heart. Fetal Diagn Ther 2017;42:161-173.

11. 11.Turan S, Turan OM, Ty-Torredes K, Harman CR, Baschat AA. Standardization of the first-trimester fetal cardiac examination using spatiotemporal image correlation with tomographic ultrasound and color Doppler imaging. Ultrasound Obstet Gynecol 2009;33:652-656.

12. Abuhamad AZ, Chaoui R. A Practical Guide to Fetal Echocardiography. Normal and abnormal hearts. Third edition. LWW, 2015.

13. Rasiah SV, Publicover M, Ewer AK, Khan KS, Kilby MD, Zamora J. A systematic review of the accuracy of first-trimester ultrasound examination for detecting major congenital heart disease. Ultrasound Obstet Gynecol 2006;28:110-116.

14. Vimpelli T, Huhtala H, Acharya G. Fetal echocardiography during routine first-trimester screening: a feasibility study in an unselected population. Prenat Diagn 2006;26:475482.

15. Clur SA, Bilardo CM. Early detection of fetal cardiac abnormalities: how effective is it and how should we manage these patients? Prenat Diagn 2014;34:1235-1245. 